\title{
An overview of commercially available teslameters for applications in modern science and industry
}

\author{
Dragana Popovic Renella, Sasa Spasic, Sasa Dimitrijevic, Marjan Blagojevic, Radivoje S Popovic
}

SENIS AG, Neuhofstrasse 5a, 6340 Baar, Switzerland

\begin{abstract}
The Hall-effect based teslameters (also called gaussmeters) are the mostly applied instruments for measuring DC and AC magnetic flux densities in modern science and industry. This paper gives an overview of commercially available teslameters at the high-end performance level. The teslameters have been evaluated by following characteristics that are published by suppliers: probe dimensions, magnetic field sensitive volume, accuracy, magnetic resolution, measurement range, frequency bandwidth, temperature coefficient sensitivity, and price/performance ratio.

The Teslameter that best matches the measurement needs in various application fields incorporates a 3-axis integrated Hall probe, analog electronics based on the spinning-current technique, an analog-to-digital converter, an embedded computer, and a touchscreen display. The 3 -axis Hall probe is a single silicon chip integrating both horizontal and vertical Hall magnetic sensors and a temperature sensor. The spinning-current eliminates most of the Hall probe offset, low-frequency noise, and the planar Hall voltage. The errors due to the Hall sensor non-linearity and the variations in the probe and electronics temperatures are eliminated by a calibration procedure. The errors due to the angular imperfections of the Hall probe are eliminated by a calibration of the sensitivity tensor of the probe. This teslameter can measure magnetic field vectors from about $100 \mathrm{nT}$ to $30 \mathrm{~T}$, with a spatial resolution of 100 $\mu \mathrm{m}$, magnetic resolution $\pm 2 \mathrm{ppm}$ of the range, accuracy $0.002 \%$ of the range, a temperature coefficient less than $5 \mathrm{ppm} /{ }^{\circ} \mathrm{C}$, and angular errors less than $0.1^{\circ}$.
\end{abstract}

\section{Section: RESEARCH PAPER}

Keywords: teslameter; gaussmeter; magnetic measurement; hall probe

Citation: Dragana Popovic Renella, Sasa Spasic, Sasa Dimitrijevic, Marjan Blagojevic, Radivoje S Popovic, An overview of commercially available teslameters for applications in modern science and industry, Acta IMEKO, vol. 6, no. 1, article 7, April 2017, identifier: IMEKO-ACTA-06 (2017)-01-07

Section Editor: Paul Regtien, The Netherlands

Received January 18, 2016; In final form March 2, 2017; Published April 2017

Copyright: (C) 2017 IMEKO. This is an open-access article distributed under the terms of the Creative Commons Attribution 3.0 License, which permits unrestricted use, distribution, and reproduction in any medium, provided the original author and source are credited

Corresponding author: Dragana Popovic Renella, e-mail: info@senis.ch

\section{INTRODUCTION}

A teslameter, also called gaussmeter, is an instrument for measuring magnetic flux density. Usually, a teslameter consists of a magnetic field probe and an electronic module. The probe converts the measured magnetic field into an electrical signal. Most teslameter probes used in modern science and industry are based on the Hall-effect [1] - the probe contains a Halleffect device [2]. The electronic module supports the operation of the probe, processes the signal of the probe, and provides the interface with the user. Modern teslameters are digital, meaning that the signal processing includes analogue-to-digital conversion and digital signal conditioning, and the user interface includes a digital display. A digital teslameter with a magnetic field probe based on a Hall-effect device is often called - in short - a Hall teslameter.

Hall teslameters are used for measuring magnetic flux densities in the magnetic field range from a few $\mu \mathrm{T}$ to about 30 $\mathrm{T}$, a frequency range from $\mathrm{DC}$ to several tens of $\mathrm{kHz}$, and a temperature range from a few degrees $\mathrm{K}$ to about $200{ }^{\circ} \mathrm{C}$ (although most of commercially available teslameters are intended for a much narrower temperature range around room temperature). The best published characteristics of modern Hall teslameters include a magnetic resolution as high as $0.1 \mathrm{ppm}$ and a magnetic field measurement accuracy of up to $50 \mathrm{ppm}$ of the measurement range. However, the measurement accuracy of a teslameter is usually strongly deteriorated by temperature variations, at AC measurement conditions, and by nonhomogeneity of the measured magnetic fields. Accurate 
measurement of inhomogeneous magnetic fields is steadily getting more important and challenging because of the long trend of miniaturization of technical systems. For example, this is so in the field of mapping of small magnets used in conjunction with magnetic sensors [3]-[5], or in the measurement of the magnetic field of the undulators of electron light sources [6]. In Chapter 2 we briefly introduce some teslameter applications in modern science and industry in order to demonstrate the importance of certain characteristics of the teslameters.

The measurement accuracy of nonhomogeneous magnetic fields is additionally deteriorated if the following conditions are not sufficiently met [7], [8]:

- Small sensitive volume of the probe, which allows for high spatial resolution of the magnetic measurement;

- Measurement of all three components of a magnetic field simultaneously at the same spot;

- Small overall dimensions of the probe, so that it can be inserted into a small space;

- Accurate spatial positioning of the probe with respect to a coordinate system;

- Accurate angular positioning of the probe with respect to a coordinate system;

- Parallelism of the sensitivity vector(s) of the probe with the axes of the coordinate system;

- No planar Hall effect.

Each magnetic measurement task requires a specific combination of the characteristics of a teslameter. Unfortunately, it is often not easy to select the most appropriate teslameter for a given application. The difficulties stem from the fact that vendors sometimes apply different terminology for the same characteristics, do not publish precise conditions of the validity of the quoted data, or simply neglect to publish some important characteristics.

In spite of these difficulties, in this paper, we will try to compare the published characteristics of the commercially available teslameters. The global teslameter market is currently served by about 50 vendors with more than 100 types of teslameters [9]. We will concentrate in this study on the highend segment of the market, with the highest-performance teslameters for a magnetic measurement range from about $1 \mu \mathrm{T}$ to $30 \mathrm{~T}$ at about room temperature. Since the high-performance teslameters are commonly available as desktop versions only, most of hand-held teslameters were not evaluated in the study. Out of the reviewed tesalemeters, 9 models are functioning with 3-axis Hall probes, and four of them incorporate the integrated Hall probes on a single chip. the other 3-axis Hall probes are hybrid probes that consist of 3 discrete Hall sensors positioned in the way that each of them measures one component of the magnetic field.

The comparison of the teslameters is organized in the following way: in Chapter 3 we first compile and compare the best published values of the most important characteristics; then, based on these data, in Chapter 4 we try to identify the teslameter type with the best combination of the characteristics, which best meets the needs for accurate magnetic measurements in modern science and industry, as introduced in Chapter 2.

\section{TESLAMETER APPLICATIONS IN MODERN SCIENCE AND INDUSTRY}

In order to better understand why some characteristics of the teslameters are important, we briefly present in this chapter some applications in modern science and industry and their requirements for high performance teslameters.

\subsection{Teslameter application in superconductive magnets}

One application in industry is the use of teslameters for the fast measuring of magnetic fields during the on-site calibration process of superconductive magnets. Figure 1 shows a SENIS low noise \& high resolution teslameter that is used for high accuracy measurements of magnetic fields up to $20 \mathrm{~T}$ in superconductive magnets of Bruker BioSpin AG [10]. For this application, the characteristics of the teslameter must include very high DC magnetic resolution (low-frequency noise), high stability (low temperature coefficient of offset and sensitivity < $\left.5 \mathrm{ppm} /{ }^{\circ} \mathrm{C}\right)$ ), high measurement accuracy better than $0.003 \%$ and high magnetic field range. Magnetic resolution and stability should be correlated. Any improvement in one of these parameters should be followed by an improvement in the other one.

\subsection{Teslameter application in undulator systems}

An important teslameter application in modern science is the measurement of the magnetic field in undulators of the electron light sources such as SwissFEL at the Paul Scherrer Institut [10]. In addition to the standard requirements, such as high accuracy and resolution and high stability, some additional features for good teslameters are required, and particularly for their Hall probes. These are two- or three-axis probes, small overall dimensions of the probe $\left(10 \times 10 \times 1.4 \mathrm{~mm}^{3}\right.$ (3-axis)), as shown in Figure 2, a flat probe that is positioned in the gap of the undulator, small and compact magnetic field sensitive

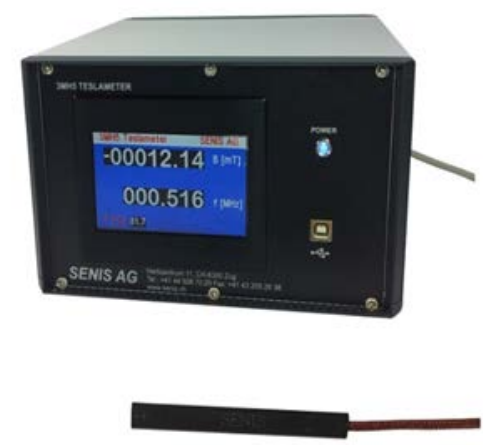

Figure 1. Low noise \& high resolution teslameter used during the calibration process of superconductive magnets [10]

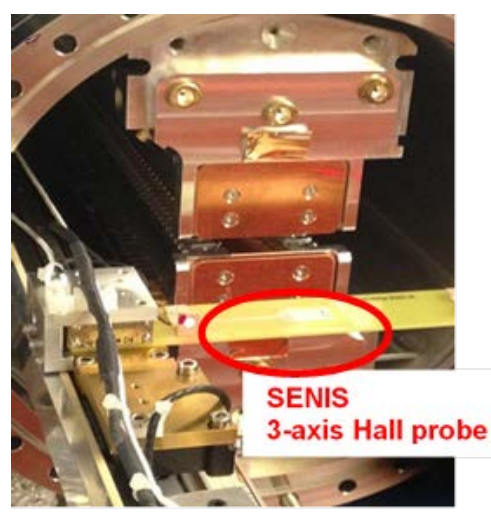

Figure 2. Flat SENIS 3-axis Hall probe used for magnetic measurements in electron light sources at PSI [10]. 
volume of the Hall probe and no planar Hall effect.

The similar application of the magnetic field mapping utilizing the high performance teslameters can be found in other National Research Laboratories throughout the world in the field of Accelerators, Light Sources, Synchrotron Radiations, etc.

\subsection{Teslameter application for magnetic field mapping around large coils}

Still another modern application requires the magnetic measurement around very large coils for current centerline determination of the superconductors, as for the ITER project, see Figure 3. Here it is important to achieve an accurate positioning of the probe with respect to a coordinate system of the measured coil ( $0.1 \mathrm{~mm}$ accuracy), measuring all three components of a magnetic field simultaneously at the same spot and small angular error of the sensitivity vectors of the probe $\left(0.1^{\circ}\right)$.

\subsection{Teslameter application for magnetic field mapping around small magnets}

Modern magnetic position and angle sensors consist of a combination of a permanent magnet and a magnetic field sensor, and they are widely used in a broad range of applications involving precision motion and control in automotive industry and sensing in industrial and consumer products. The characteristics of such magnets that are parts of positioning and angle magnetic sensors must be carefully controlled. This is usually done with a magnetic field mapper that combines a precise motion control unit and a high precision teslameter. The SENIS magnetic field mapper measures all three components of the magnetic field at precisely defined points around permanent magnets (from $1 \mathrm{~m}$ to $50 \mathrm{~cm}$ ) with the magnetic field measurement range from $100 \mu \mathrm{T}$ to $3 \mathrm{~T}$. Among others, Bomatec AG, Figure 4, utilizes the SENIS Magnetic Field Mapping System for quality control of permanent magnets [10].

The University of Buffalo [11] performs model validation and material/device characterization using the SENIS magnetic field mapper, which enables the measurement of 3D magnetic field distribution for arbitrary material/component geometries with $10 \mu \mathrm{m}$ spatial resolution, see Figure 5 .

\section{COMPARISON OF TESLAMETERS CHARACTERISTICS}

In each of the following tables we list three to five teslameters with the following best characteristics: 1) probe dimensions, size of the magnetic field sensitive volume, and probe angular errors; 2) magnetic resolution; 3) magnetic field measurement accuracy; 4) measurement range; 5) frequency

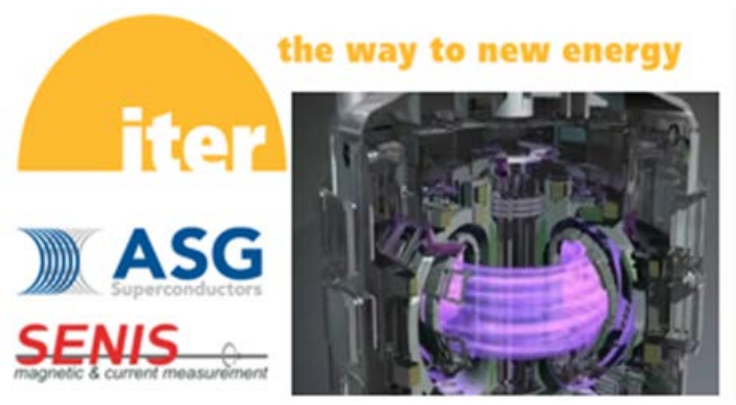

Figure 3. SENIS low-noise \& high-accuracy magnetic field measurement system is used for the ITER Project. ITER shall demonstrate the scientific and technological feasibility of fusion energy on an industrial scale [10].

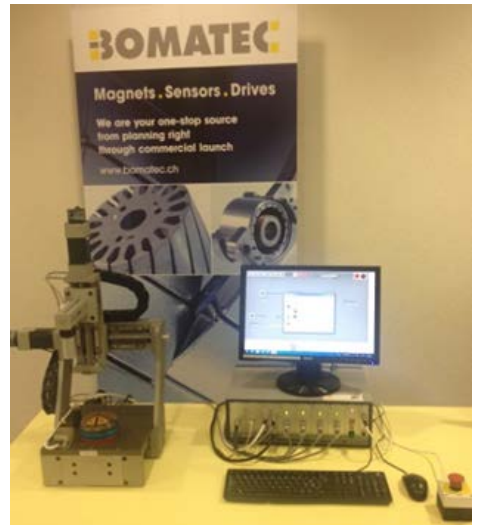

Figure 4. Bomatec AG utilizes the SENIS Magnetic Field Mapping System for quality control of permanent magnets.

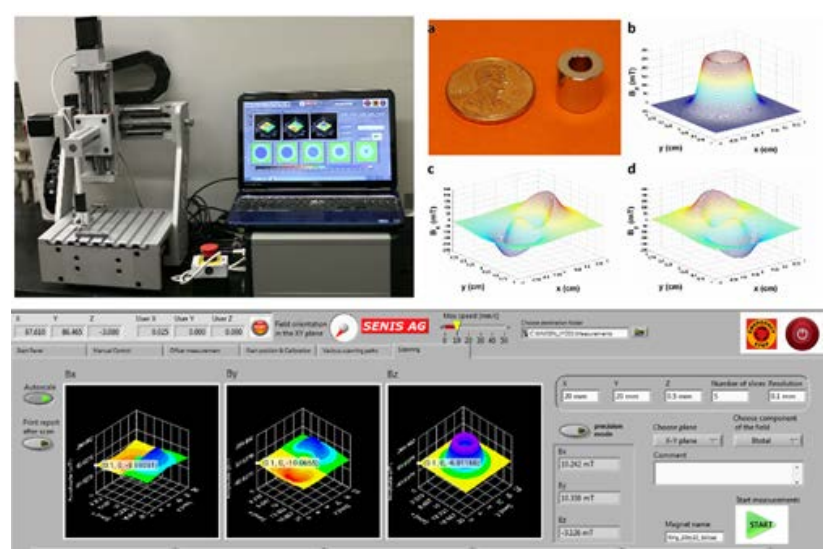

Figure 5. SENIS 3D Magnetic Field Mapper for model validation and material/device characterization at University of Buffalo, NY [11].

bandwidth and 6) temperature coefficient of sensitivity.

\subsection{Probe dimensions, magnetic field sensitive volume and probe angular error}

For measuring non-homogenous magnetic fields the following characteristics are crucial: small overall dimension of the probe, measuring all three components of the magnetic field at the same spot (i.e. small magnetic field sensitive volume (MFSV) of the probe); and small angular errors of the probe (orthogonality error of the Hall devices within the probe). In Table 1 the three teslameters that best meet the required needs are presented.

Many vendors of teslameters do not quote exact dimensions of the magnetic sensitive volume nor the angular error of their Hall probes. The SENIS integrated 3 -axis Hall probe is described in [16].

\subsection{Comparison of the magnetic field measurement accuracy}

The magnetic field measurement accuracy of the teslameter is defined as the maximum difference between the actual measured magnetic flux density and that given by the teslameter. Usually, the accuracy is expressed in percentage of the measurement range. In Table 2 we list five teslameters with the best magnetic field measurement accuracy.

\subsection{Comparison of the magnetic resolution}

Resolution of the teslameter is the smallest detectable change of the magnetic flux density that can be revealed by the teslameter. The resolution is limited by the noise and depends on the frequency band of interest. Vendors usually quote both 
Table 1. Comparison of probe dimensions, magnetic field sensitive volume and angular errors of the probe.

\begin{tabular}{|c|c|}
\hline Published probe characteristics & Provider \\
\hline $\begin{array}{l}\text { - } \quad \text { Fully integrated 3-Axis Hall probe; } \\
\text { Various and customizable dimensions } \\
\text { (Width } \times \text { Thickness), e.g.: } \\
\text { Probe C: } 4.0 \mathrm{~mm} \times 0.9 \mathrm{~mm} \\
\text { Probe K: } 2.0 \mathrm{~mm} \times 0.5 \mathrm{~mm} \\
\text { Thinnest probe: } 0.25 \mathrm{~mm} \\
\text { MFSV distance from the tip of the } \\
\text { probe: } 0.15 \mathrm{~mm} \\
\text { - } \\
\text { MFSV: } 0.1 \times 0.1 \times 0.1 \mathrm{~mm}^{3} \\
\text { Orthogonality error (calibrated): } 0.1^{\circ}\end{array}$ & SENIS AG [12] \\
\hline $\begin{array}{l}\text { - } \quad \text { Hybrid 3-axis Hall probe; } \\
\text { Probe dimensions }(\mathrm{W} \times \mathrm{Th}): 5 \mathrm{~mm} \times 2 \\
\mathrm{~mm} \\
\text { - } \quad \text { FSV distance from the tip of the } \\
\text { probe: } 3 \mathrm{~mm} \\
\text { - } \quad \text { MFSV: } 0.1 \times 0.1 \times 0.1 \mathrm{~mm}^{3} \\
\text { Orthogonality error (calibrated) }\end{array}$ & COLIY [13] \\
\hline $\begin{array}{l}\text { - Applies SENIS Hall probes, among } \\
\text { others [14] }\end{array}$ & METROLAB [15] \\
\hline
\end{tabular}

Table 2. Comparison of the magnetic field measurement accuracy.

\begin{tabular}{l|l}
\hline $\begin{array}{l}\text { Published magnetic field accuracy } \\
\text { DC-accuracy of range }\end{array}$ & Provider \\
\hline \hline $0.002 \%$ & SENIS [12] \\
Integrated 3-axis Hall probe & \\
\hline $0.005 \%$ Single channel & Projekt Elektronik [17] \\
$0.5 \%$ 3-channel & \\
\hline $0.05 \%$ Single channel & Lake Shore [18] \\
$0.1 \%$ 3-channel & \\
\hline \hline $0.01 \%$ Single channel only & Group3 [19] \\
\hline \hline $0.1 \%$ 3-channel & FW Bell [20] \\
\hline
\end{tabular}

DC resolution and AC resolution, but they rarely specify the related bandwidth. Though, one vendor [12] expresses DC resolution by quoting "Offset fluctuation \& drift" over the period of time $10 \mathrm{~s}$ (or $100 \mathrm{~s}$ ) with the sampling rate $20 / \mathrm{s}$ which corresponds to a frequency range from $0.1 \mathrm{~Hz}$ (or 0.01 $\mathrm{Hz}$ ) to $10 \mathrm{~Hz}$. Table 3 gives a comparison of the five teslameters with the best magnetic resolution.

\subsection{Comparison of the measurement range and frequency bandwidth}

Hall-effect-based teslameters are applied for measuring DC and $\mathrm{AC}$ magnetic flux densities in the range from about $1 \mu \mathrm{T}$ to about $30 \mathrm{~T}$. The frequency bandwidth characterizes how well a magnetometer tracks rapid changes in magnetic field. Some vendors do not specify whether or not there is inductive pick-

Table 3. Comparison of the magnetic resolution.

\begin{tabular}{l|l}
\hline Published magnetic resolution & Provider \\
\hline \hline $0.1 \mu \mathrm{T}$ & SENIS [12] \\
\hline \hline $0.1 \mu \mathrm{T}$ & Group3 [19] \\
\hline \hline $0.1 \mu \mathrm{T}$ & Projekt Elektronik [17] \\
\hline \hline $0.1 \mathrm{mT}$ & FW Bell [20] \\
NOTE: for the range of 30 $\mu \mathrm{T}, \mathrm{a}$ & \\
resolution of 0.1 nT available & \\
\hline $\begin{array}{l}0.1 \mathrm{mT} \text { NOTE: for the range of } 3.5 \mu \mathrm{T}, \mathrm{a} \\
\text { resolution of } 2 \mathrm{nT} \text { available }\end{array}$ & Lake Shore [18] \\
\hline
\end{tabular}

up in the connections of their Hall probes at the upper part of the broad frequency range they quote, which makes the comparison difficult. Table 4 gives a comparison of the measurement ranges and frequency bandwidth of the five best teslameters.

\subsection{Comparison of the temperature coefficient of sensitivity}

The thermal stability of the teslameter is the dependence of the measurement on temperature. It is given as a temperature coefficient of sensitivity in ppm per degree Celsius. Table 5 gives a comparison of the temperature coefficient sensitivity of the five best teslameters.

\subsection{Summary of the teslameters performances and prices}

By comparing the teslameters by the above characteristics we found that there are two providers offering 3-axis Hall probes with very small magnetic field sensitive volume and small probe angular errors. Two providers guarantee very high DC-accuracy of the measurement range. Three providers offer high magnetic resolution for their Hall-effect based 3-axis teslameters. The temperature coefficient of sensitivity is better than $30 \mathrm{ppm}$ in three cases.

Last but not least is the price/performance ratio of the evaluated teslameters. Whereas 1-axis high-end teslameters range from $3 \mathrm{k} \$-6 \mathrm{k} \$$, the 2 -axis are between $5 \mathrm{k} \$-10 \mathrm{k} \$$, and the 3 -axis teslameters are in the price segment of $14 \mathrm{k} \$-20 \mathrm{k} \$$.

\section{THE TESLAMETER WITH THE BEST COMBINATION OF PERFORMANCES}

In four out of five tables, the teslameter of SENIS [12] appears at the top of the list. In summary, this new teslameter can measure magnetic field vectors of a magnitude from about $1 \mu \mathrm{T}$ to $30 \mathrm{~T}$, with an angular error less than $0.1^{\circ}$, spatial resolution $100 \mu \mathrm{m}$, magnetic resolution $\pm 2 \mathrm{ppm}$ of the range, with an accuracy of $0.002 \%$ of the range, and a temperature coefficient less than $5 \mathrm{ppm} /{ }^{\circ} \mathrm{C}$. We present below some details of this teslameter [21].

\subsection{Novel Integrated Three-axis Hall probe}

Accurate measurement of highly non-homogeneous magnetic fields requires a small and compact sensitive volume of the Hall probe and measuring all three components of a magnetic field simultaneously at the same spot. An early version

Table 4. Comparison of the measurement ranges and frequency bandwidth

\begin{tabular}{l|l|l}
\hline $\begin{array}{l}\text { Published } \\
\text { measurement } \\
\text { range }\end{array}$ & $\begin{array}{l}\text { Published frequency } \\
\text { bandwidth (per axis) }\end{array}$ & Provider \\
\hline \hline $1 \mu \mathrm{T}-30 \mathrm{~T}$ & DC-75 kHz & SENIS [12] \\
\hline \hline $1 \mu \mathrm{T}-30 \mathrm{~T}$ & DC-50 kHz & FW Bell [20] \\
\hline \hline $1 \mu \mathrm{T}-30 \mathrm{~T}$ & $\mathrm{DC}-20 \mathrm{kHz}$ & Lake Shore [18] \\
\hline $0.3 \mathrm{~T}-3 \mathrm{~T}$ & $\mathrm{DC}-3 \mathrm{kHz}$ & Group3 [19] \\
\hline \hline $20 \mathrm{mT}-2 \mathrm{~T}$ & $\mathrm{DC}-1 \mathrm{kHz}$ & Projekt Elektronik [17] \\
\hline
\end{tabular}

Table 5. Comparison of the temperature coefficient sensitivity.

\begin{tabular}{l|l}
\hline Published temperature coefficient sensitivity & Provider \\
\hline \hline $3 \mathrm{ppm}$ & Projekt Elektronik [17] \\
\hline \hline $5 \mathrm{ppm}$ & SENIS [12] \\
\hline $10 \mathrm{ppm}$ & Group3 [19] \\
\hline $30 \mathrm{ppm}$ & Lake Shore [18] \\
\hline $200 \mathrm{ppm}$ & FW Bell [20] \\
\hline
\end{tabular}


of the Senis integrated 3-axis Hall probe [16] fulfilled well these requirements, but the residual flicker noise of this probe limited the measurement accuracy to about $0.1 \%$. SENIS recently developed a new integrated 3-axis Hall probe with much better performance, Figure 6 .

The new Senis 3-axis Hall probe incorporates several horizontal and vertical Hall devices, monolithically integrated on a single silicon CMOS chip. Both horizontal and vertical Hall devices feature high magnetic sensitivity (higher than 0.04 $\mathrm{V} / \mathrm{VT}$ ) and low flicker noise (the corner frequency lower than 2 $\mathrm{kHz}$ ) [22]. For each measurement axis, eight equal parallelconnected Hall devices are used. This allows reducing the noise equivalent magnetic field spectral density of the thermal noise of each group of Hall devices to about $40 \mathrm{nT} / \sqrt{\mathrm{Hz}}$. All 24 Hall devices occupy an area of only $100 \mu \mathrm{m} \times 100 \mu \mathrm{m}$. A temperature sensor, which is also integrated on the Hall probe chip, enables an efficient temperature compensation of the influences of the probe temperature.

The electronic module drives the Hall devices in the probe according to an optimized spinning-current method. The spinning-current method is well described in Appendix A of [16]: the Hall device is connected to a biasing source and an output circuit via a group of switches. The switches are turned on and off so that the diagonally situated terminals of the Hall device are periodically commutated and alternatively used as the current (input) and the sense (output) contacts. Thus, the bias current virtually rotates in the device for $90^{\circ}$ back and forth (therefore, the term "spinning current"). The Hall voltage rotates with the biasing current and appears at the output with a sign that depends only on the orientation of the magnetic field B. If the magnetic field does not vary much during a switching period, then the Hall voltage is quasi-DC. On the other hand, the offset voltage at the output has an opposite sign during each phase of the spinning current. Therefore, the offset voltage appears as an AC signal with the switching frequency and can be filtered out from the output voltage.

The resulting modulation-demodulation process of the Hall voltages eliminates the offset and the flicker noise of both the Hall devices and the amplifiers of the Hall signal; and it also eliminates the planar Hall voltage. After analog-to-digital conversion of the "clean" and amplified Hall signals with up to 22 significant bits, the Hall signals are further processed by an embedded computer. The digital processing includes filtering, linearization, and corrections of temperature dependence. The cancellation of the temperature influence is based on a novel calibration procedure that takes into account both the temperature of the probe and the temperature of the electronics module. The calibration process also includes the precise

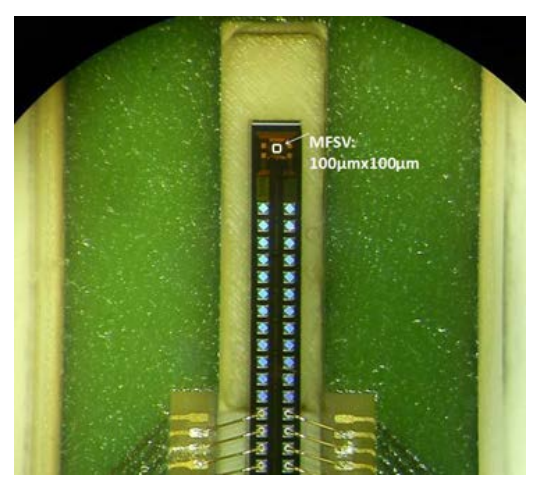

Figure 6. SENIS 3D Hall Probe. Spacial Resolution:100 $\mu \mathrm{m} \times 100 \mu \mathrm{m}$ The probe is packaged in alumina ceramics. Probe dimensions $(\mathrm{W} \times \mathrm{Th} \times \mathrm{L})$ in $\mathrm{mm}: 6.0 \times 1.6 \times 14.5$. measurement of the nine components of the sensitivity tensor of the probe [23], which allows the elimination of the errors that might be caused by the angular tolerances of the Hall probe. The interface with the user is provided by a touch-screen display.

\subsection{Latest measurement results of the novel teslameter}

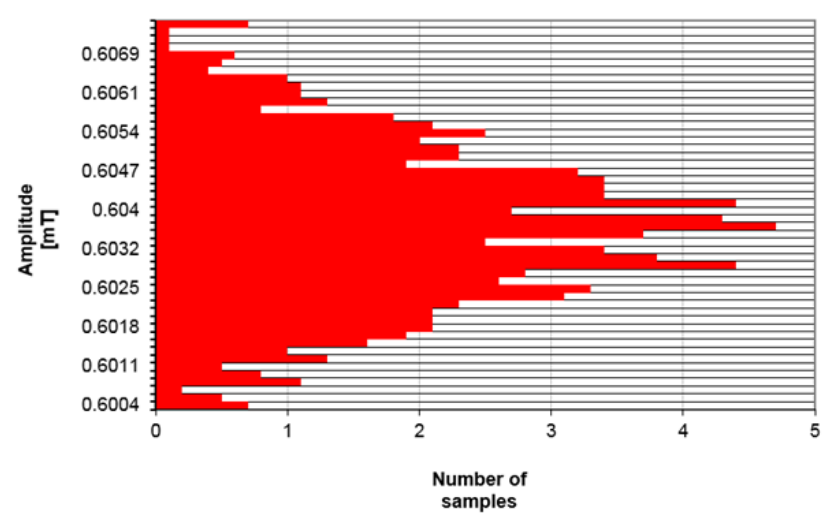

Figure 7. Histogram of the noise-equivalent magnetic field of the $3 \mathrm{MH} 5$ SENIS' Teslameter with the low-noise hybrid Hall probe, in the magnetic measurement range $2 \mathrm{~T}$ and a frequency range from $0.1 \mathrm{~Hz}$ to $10 \mathrm{~Hz}$. The calculated standard deviation is $1.2 \mu \mathrm{T}$ and the six sigma peak-to-peak noise is $7.2 \mu \mathrm{T}$, which corresponds to the width of the histogram. At DC measurements (integration time $1 \mathrm{~s}$ ) the teslameter has a peak-to-peak resolution of about $1 \mathrm{ppm}$ of the measurement range.

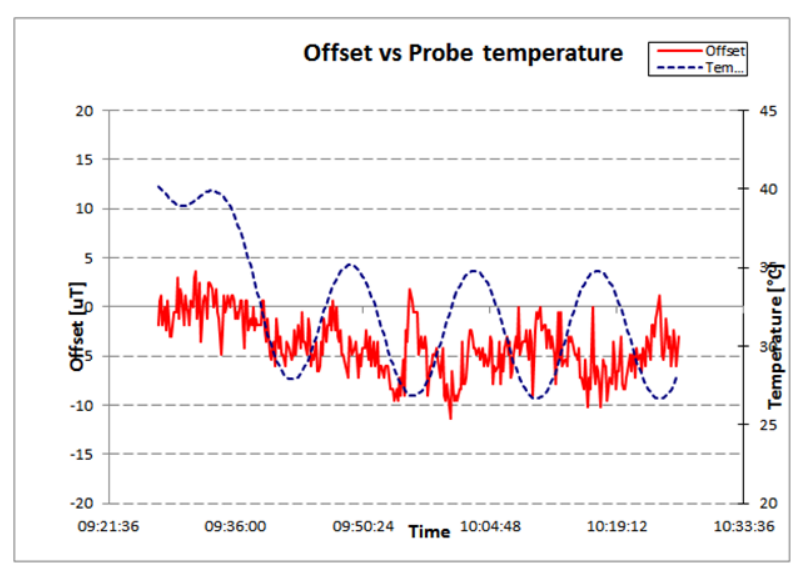

Figure 8. Illustrating offset stability of the 3MH5 SENIS' Teslameter. Solid red line: offset, left scale; dashed blue line: probe temperature, right scale. The figure shows that, at room temperature, the offset fluctuations are not correlated with temperature.

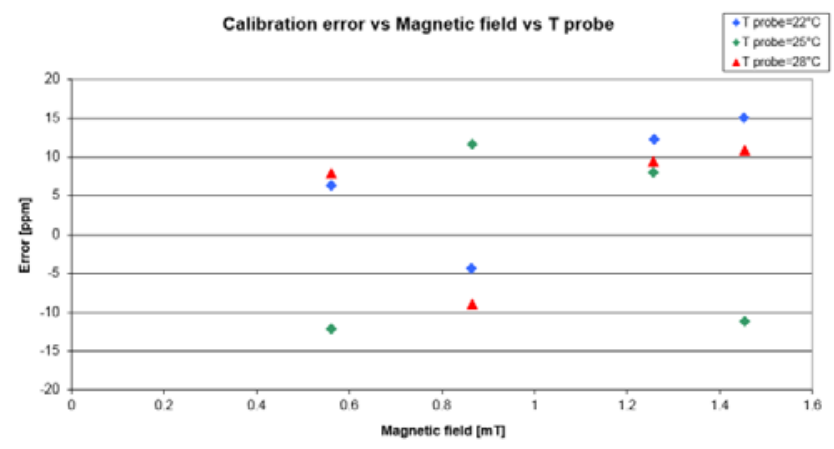

Figure 9. The measurement error of the $3 \mathrm{MH} 5$ SENIS' Teslameter in the measurement range $2 \mathrm{~T}$. If the room temperature varies within $+/-3{ }^{\circ} \mathrm{C}$ around $22{ }^{\circ} \mathrm{C}$, the measurement error is within $+/-15 \mathrm{ppm}$ (parts per million) of the measured value. 


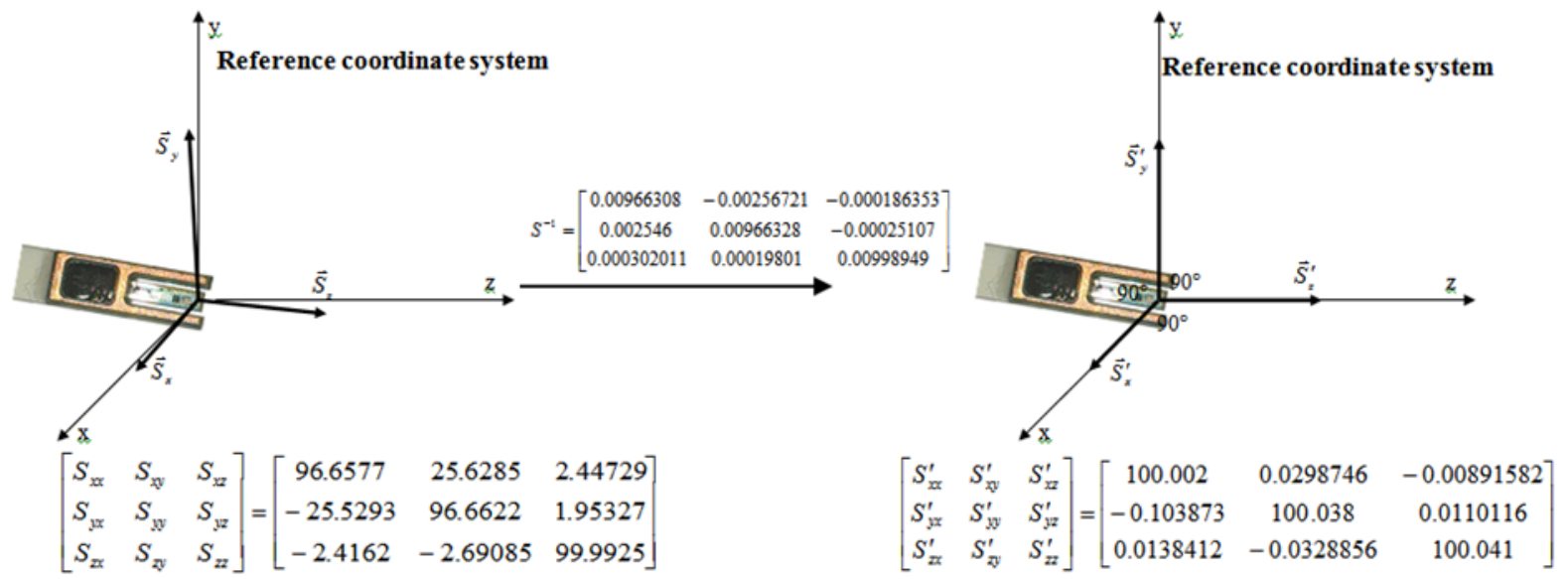

Figure 10. Illustrating the correction of the angular errors of a 3-axis integrated Hall probe [23]. In order to demonstrate the efficiency of the calibration method, the probe was rotated with respect to the z-axis for about $15^{\circ}$. Left: the "raw" sensitivity matrix (before calibration); middle: the inversed "raw" sensitivity matrix; right: the corrected sensitivity matrix, which is an almost identity matrix, with negligible off-diagonal terms. The related angular errors are shown in Table 6.

Table 6. Angular errors of the 3-axis integrated Hall probe with the sensitivity matrices shown in Figure 5. Notation: $X_{r}$ - Roll of x sensor; $X_{t}$ Tilt of $\mathrm{x}$ sensor; $y_{p}$ - Pitch of y sensor; $y_{r}$ - Roll of y sensor; $z_{p}-$ Pitch of $z$ sensor; $Z_{t}$ - Tilt of $z$ sensor. In spite of the deliberately produced big initial error $X_{r}$ and $y_{r}$, after the calibration of the probe, all angular errors are reduced to less than $0.1^{\circ}$.

\begin{tabular}{|c|c|c|c|c|c|c|}
\hline & $X_{r}$ & $X_{t}$ & $y_{p}$ & $y_{r}$ & $Z_{p}$ & $Z_{t}$ \\
\hline $\begin{array}{c}\text { Before } \\
\text { calibration }\end{array}$ & $14.85^{\circ}$ & $1.45^{\circ}$ & $1.16^{\circ}$ & $-14.79^{\circ}$ & $-1.54^{\circ}$ & $-1.38^{\circ}$ \\
\hline $\begin{array}{c}\text { After } \\
\text { calibration }\end{array}$ & $0.017^{\circ}$ & $-0.005^{\circ}$ & $0.006^{\circ}$ & $-0.059^{\circ}$ & $0.018^{\circ}$ & $0.008^{\circ}$ \\
\hline
\end{tabular}

First measurement results of the novel teslameter have been published [7], [8]. Here we present the latest characterization results regarding magnetic resolution, measurement accuracy, temperature compensation and orthogonality error - see Figures $7-10$ and Table 6.

\section{CONCLUSIONS}

We compared the published performance of the best, mostly 3-axis, Hall-effect teslameters that are commercially available on the global market. The comparison was based on the following characteristics: probe dimensions, magnetic field sensitive volume, probe angular errors, magnetic resolution, magnetic field measurement accuracy, magnetic measurement range, frequency bandwidth, and temperature coefficient of sensitivity. The novel SENIS 3-axis teslameter with integrated Hall probe seems to match best the demanding measurement needs in various applications in modern science and industry.

\section{REFERENCES}

[1] E. H. Hall, "On a new action of magnetism on a permanent electric current", Am. J. Sci. series 3, 20 161-186, 1880

[2] R.S. Popovic, "Hall Effect Devices", 2nd ed., IOP Publishing Bristol and Philadelphia, 2004.

[3] M. Blagojevic, N. Markovic, and R. S. Popovic, "Testing the Homogeneity of Magnets for Rotary Position Sensors",
INTERMAG IEEE International Magnetics Conference May 4 8, 2014, Dresden, Germany.

[4] D. Popovic Renella and S. Spasic, "High-Accuracy 3-axis Teslameter and its application in the 3D Mapping of Magnetic Fields", UK Magnetic Society International Event on Magnetic Materials and Applications, Oct. 28-29, 2014, Lucerne, Switzerland.

[5] S. Spasic, I.J. Walker, M. Blagojevic, N. Markovic, "Integrating Magnetic Field Mapping and Coordinate Measurement", Magnetics 2015 Conference, Jan. 21-22, 2015, Orlando FL, USA

[6] S. Sanfilippo, "Hall probes: physics and application to magnetometry", PSI Switzerland, 2011.

[7] D. Popovic Renella, S. Dimitrijevic, S. Spasic and R.S. Popovic, "High-Accuracy Teslameter with Thin Three-Axis Hall Probe", Proceedings of 20th IMEKO TC4 International Symposium and 18th International Workshop on ADC Modelling and Testing Research on Electric and Electronic Measurement for the Economic Upturn, Benevento, Italy, September 15-17, 2014.

[8] D. Popovic Renella, S. Dimitrijevic, S. Spasic, R.S. Popovic, "High-accuracy teslameter with thin high-resolution three-axis Hall probe", Measurement, Elsevier, ISSN: 0263-2241, In Press, Available online 15 September 2015.

[9] S. Spasic, Z.Mitrovic, "Teslameter-Market Analysis", unpublished, SENIS internal document, February 2015.

[10] Teslameters in customer applications: http://www.senis.ch/products/applications/references

[11] http://www.cbe.buffalo.edu/people/full time/e furlanires CM MApp.php

[12] www.senis.ch

[13] www.coliy.com

[14] Ph. Keller, "Recent advances in Hall Magnetometers", Magnetics Magazine 2015: $\quad$ http://www.metrolab.com/wpcontent/uploads/2015/07/THM1176 tech note-published-inMagnetics-Technology-International.pdf

[15] www.metrolab.com

[16] D. Popovic, S. Dimitrijevic, M. Blagojevic, P. Kejik, E. Schurig, and R. Popovic, "Three-Axis Teslameter With Integrated Hall Probe", IEEE Trans. on Instrum. and Meas., vol. 56, no. 4, August 2007

[17] www.projekt-elektronik.com

[18] www.lakeshore.com

[19] www.group3technology.com

[20] www.fwbell.com

[21] D.R. Popovic Renella, R.S. Popovic, "Hall magnetic transducers with NMR-like resolution", IMMW 16 - International Magnetic Measurement Workshop, Bad Zurzach, Switzerland, 26-29 October 2009. 
[22] R.S. Popovic, "High Resolution Hall Magnetic Sensors", MIEL May 12-14, 2014, Belgrade, Serbia.

[23] S. Spasic, M. Blagojevic, N. Markovic, Z. Mitrovic2, L. Popovic and R.S. Popovic, "Mapping Permanent Magnets with High Angular Accuracy", AMA Sensor Conference, Nuremberg, 19 21 May 2015. 